\title{
A multiline spectrometer for the analysis of solar atmospheric oscillations and flows at the VTT, Tenerife
}

\author{
J. Staiger ${ }^{1}$ \\ Kiepenheuer-Institut für Sonnenphysik, Schöneckstrasse 6, 79104 Freiburg, Germany \\ e-mail: joe@kis.uni-freiburg.de
}

Received 16 June 2011 / Accepted 15 September 2011

\begin{abstract}
Context. Despite longstanding observational efforts, the origins of the chromospheric temperature rise and the coronal heating are still not well understood. There is reason to believe that the limitations of existing observational devices might be contributing to this lack of experimental evidence.

Aims. We intended to develop a multiline spectrometer capable of observing velocity fields simultaneously at more height levels of the solar atmosphere than previously possible. System design and handling would be optimized for the 3D-analysis of atmospheric waves and flows.

Methods. The number of optical components was kept to a minimum in order to achieve high optical throughput and short scanning times. A new type of bandpass preselection unit was developed. We successfully tested this Fabry-Perot based multiline device at the Vacuum Tower Telescope (VTT).

Results. During a proof-of-concept run we were able to observe 16 spectral lines at a cadence of $60 \mathrm{~s}$ sustained over several hours. The field of view was 100-by-100 arcsecs. Multiple diagnostic diagrams from closely spaced height levels were derived.

Conclusions. A new instrument of this type will be installed permantently at the VTT. We expect to be able to collect new 3Dinformation about atmospheric waves and flows.
\end{abstract}

Key words. techniques: spectroscopic - instrumentation: interferometers - Sun: atmosphere - waves - Sun: helioseismology

\section{Introduction}

Etalon-based spectrometers have become primary observational tools in solar surface spectroscopy. Future large telescopes like ATST or EST are expected to have observational devices of this type available from the beginning. Multiline capabilities are seen as an important operational feature in this context.

We are currently developing a multiline spectrometer for the Vacuum Tower Telescope at Tenerife optimized for the analysis of wave propagation in the solar atmosphere. By implementing a new type of fast spectral-band changing mechanism, we were able to demonstrate the capability of such an instrument to allow for a large number of spectral lines to be observed in a quasisimultaneous mode. Compared to existing devices a fourfold increase in vertical height resolution was achieved. Based on these results the decision has been made to install a new instrument of this type permanently at the VTT. The spectroscopic components have already been integrated into the mechanical setup and are currently undergoing final performance tests. The operating software was integrated into the VTT control structures in 2010. After the successful completition of the comissioning process, which is set for 2012, the spectrometer will go into scientific service.

Under the assumption that the GREGOR telescope will become fully operational in 2012, the majority of current VTT scientific activity can be expected to migrate towards the new telescope. This will free the VTT for investigations requiring extended recording times. The multiline instrument is foreseen to take a significant share of these capacities. This will allow for long duration helioseismologic observations of the solar atmosphere currently unavailable elsewhere. In the future the instrument will be further upgraded to a fast flare response mode optimized for the observation of flare related events.

We describe here the layout of the new instrument and its handling properties. We give an overview of the software structure and performance tests.

\section{Fabry-Perot spectroscopy}

\subsection{Overview}

First spectroscopic 2D-imaging experiments with tunable Etalon systems go back to the PEPSIOS instrument (Mack et al. 1963). Interferometric devices for solar type applications were first designed by Ramsay et al. (1970) and Loughhead et al. (1978). Tandem solutions coupling single etalons with birefringent filters were pioneered by Cavallini et al. (1979) and Bonaccini et al. (1989). Similar developments were initiated in 1986 by the Universitätssternwarte Göttingen at the VTT (Bendlin et al. 1992). With piezo-driven, servo-stabilized etalon systems becoming affordable, these hybrid systems would later be replaced by purely interferometric designs. Multiple etalon systems are now common with most major solar telescopes:

- TESOS at VTT, Tenerife (Kentischer 1998);

- IBIS at DDT, Sunspot NM (Cavallini 2006);

- CRISP at SST, La Palma (Scharmer 2008);

- GFPI at GREGOR, Tenerife (Puschmann 2006). 


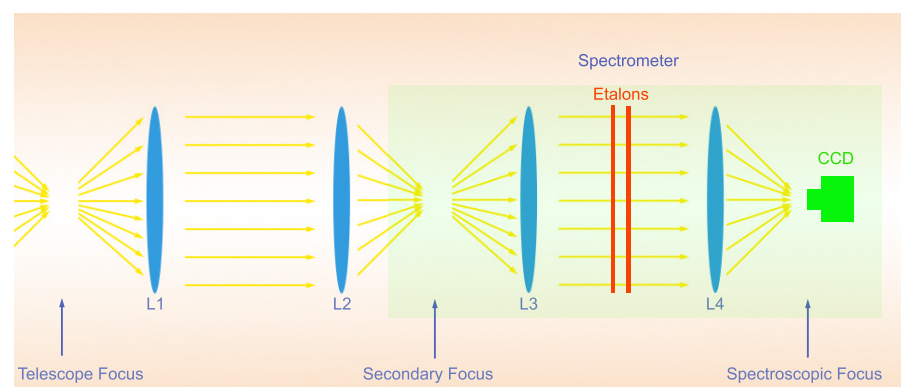

Fig. 1. Optical layout: collimated-beam configuration.

\subsection{Multiline objectives}

A consistent observational picture that would explain the energy transfer between the solar surface and the hot outer layers of the solar atmosphere does not currently exist. Tunneling of evanescent P-modes through the photomertric barrier and subsequent shock formation or magnetic mode conversions have long been suggested as possibilities for heating up the solar chromosphere and thus creating the observed steep temperature rise.

Despite longstanding efforts, no convincing experimental evidence has been established yet. Only recently does the detection of spectroscopic signatures of chromospheric shockfronts seem to support such a picture (Vecchio et al. 2009; Cauzzi et al. 2008), but neither the temporal resolution of these measurements nor the achieved layer separation would allow a final conclusion about energy net flux issues or the details of process lifecycles.

There is reason to believe that any spectroscopic instrument offering enhanced height resolution and cadence capabilities would improve this situation and might contribute to a better understanding of the atmospheric heating process.

\section{Spectrometer optical layout}

Two types of optical layouts are common for dual-etalon Fabry-Perot based spectrometers: telecentric-beam and collimated-beam configurations. Telecentric systems offer small wavelength gradients across the spectroscopic focal plane as a primary advantage. Drawbacks include a higher number of optical surfaces and spectral resolution issues that are setting upper limits to the size of the field-of-view. Collimated-beam type instruments, on the other hand, are easy to adjust and allow for high optical throughput and short exposure times. Large fieldsof-view are possible without penalties to the spectral resolution. These benefits are in turn adversely balanced by a strong nonlinear wavelength gradient spreading radially outwards from the optical axis.

We have opted for the colllimated-beam layout for our instrument as the adverse effects of the nonlinear dispersion can easily be handled by software. Figure 1 outlines the optical setup. The primary image of the sun as created within the telescope's focal plane (left) will be mapped towards the secondary focus (center) by the transfer optics components L1 and L2. Reimaging by lenses L3 and L4 will create the final spectroscopic image as recorded by a CCD camera (right). The two Fabry-Perot etalons (red) are placed within the parallel beam area between lenses L3 and L4.

Extended knowledge about the optical performance of collimated systems (e.g. IBIS) has already been collected and is readily available (Cavallini 2006). A detailed performance analysis of telecentric-versus-collimated beam systems has recently been published by Righini et al. (2010).

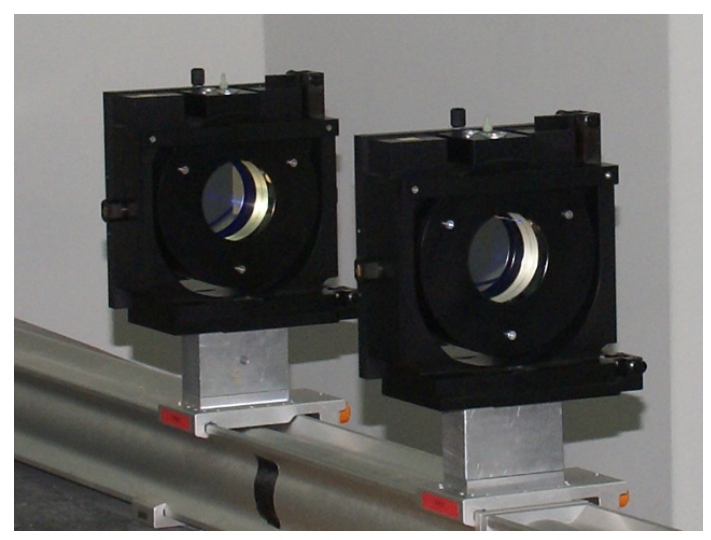

Fig. 2. Dual Etalons at the VTT.

\section{Hardware components}

\subsection{Etalons}

Two Fabry-Perot Etalons of IC-Optical Systems Ltd., Beckenham, UK with clear apertures of $70 \mathrm{~mm}$ were used for the VTT tests (Fig. 2).

Plate spacings of $1.1 \mathrm{~mm}$ and $1.4 \mathrm{~mm}$ resulted in FSR values of $0.19 \mathrm{~nm}$ and $0.25 \mathrm{~nm}$ respectively at a wavelength of $500 \mathrm{~nm}$ (FSR: free spectral range). Coating reflectivity stood at 86.3 and finesse values at 46 with a surface flatness of $\lambda / 200$. Triple-piezo stacks served as actuators for plate tilting and shifting. With the help of glass reference capacitators and closed loop procedures the wavelength can be kept stable to appr. $0.01 \mathrm{pc}$ of the free spectral range. Spectral transmission ranged from $510 \mathrm{~nm}$ to $860 \mathrm{~nm}$. Plate controller access is via RS-232 interfacing. Information exchange and action triggering are overseen by a set of ASCII firmware commands.

The etalons were part of the GFPI-instrument formerly attached to the VTT, which since then has been relocated to the GREGOR telescope (GFPI: Göttingen Fabry-Perot Interferometer).

\subsection{Computing}

Etalon activities and data recording are coordinated by an offthe-shelf personal computer running under Windows XP. Piezo controllers are addressed via 8-port USB-to-serial interfaces (Type: ET400, Quatech Corp.). All code has been written in $\mathrm{C}++$ from within an MS Visual Studio development environment (C)1987-2002 Microsoft Corporation.). A 100 GBit ethernet link couples to the VTTs telescope control software.

\section{3. $C C D$ imaging}

Spectroscopic images are recorded by a DALSA Megapixel CCD camera (Type: 1M30). At 14 micron pixel size and a filling factor of 100 , the recording area is $1.43 \mathrm{~cm}$ square. A transfer pipeline operating at $40 \mathrm{MHz}$ feeds data into a framegrabber board (MATROX Meteor Digital II), achieving frame rates of up to $30 \mathrm{images/s}$ at 12 bit resolution. Camera functionality is controlled by a set of ASCII commands via RS232-interface.

Narrowband spectroscopic images are mapped to the chip area on a scale of $6.96 \mathrm{arcsec} / \mathrm{mm}$ equaling a pixel spacing of $0.1 \mathrm{arcsec}$. This will be reduced to a value of $0.2 \mathrm{arcsec}$ by means of 2-by-2-binning, which fits to the VTTs theoretical resolution limit of 0.2 arcsec. 


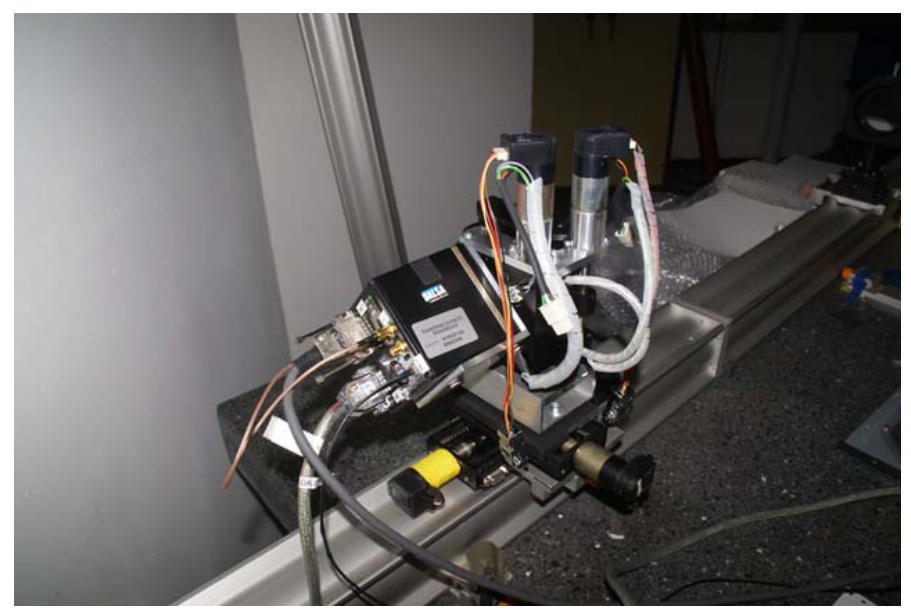

Fig. 3. DALSA CCD camera with 4-axis mount.

At a size of 100-by-100 arcsec the field of view is among the largest by current spectrometer standards.

\subsection{Image data storage}

Image frames are sampled and stored scan-wise as raw sequential binary files at two bytes/pixel. A tree-like directory structure consists of dedicated subdirectories for individual spectral lines during multiline operations. Information about scan parameters, solar positioning, run timing, telescope status, seeing quality, and sky brightness is to be stored into an equivalent, but separate directory tree. Data are stored in external USB 2.0 harddisks.

\subsection{Image alignment}

The correct placement and adjustment of the CCD's detector area within the image plane is accomplished by a computercontrolled four-axis mount (Fig. 3). A linear stage drive aligned along the optical axis (lower left to upper right) allows placing the detector surface exactly within the spectroscopic focal plane. Lateral camera positioning is controlled by a dual-axis Cartesian drive. A rotational drive will compensate for varying image axis tilts as caused by the daytime variations of the coelostat's azimutal position.

\section{Fast bandpass preselection}

\subsection{Overview}

Figure 4 shows the transmission curves for the two etalons used during the experiments. At an input wavelength of $500 \mathrm{~nm}$, the plate spacings of $1.1 \mathrm{~mm}$ and $1.4 \mathrm{~mm}$ will result in transmission peak spacings of $0.12 \mathrm{~nm}$ and $0.15 \mathrm{~nm}$, respectively.

The combined transmission curve of both etalons as arranged in a tandem configuration is shown in Fig. 5. A narrowband interference prefilter will single out a peak (e.g. center peak), which in turn will be used as the spectroscopic probe to scan an absorption line (Fig. 6). The scanning process will be accomplished by piezo-driven synchronous variation in the etalon's plate spacings.

\subsection{Filter matrix shifter}

Operating a spectrometer in multiline mode requires the instrument to be switched between different wavelength bands

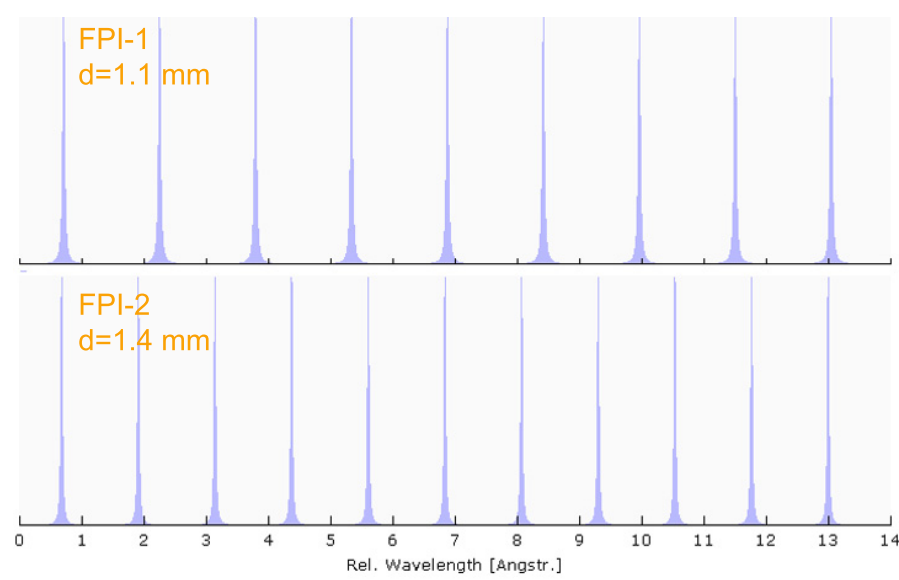

Fig. 4. Top: etalon 1 transmission, $d=1.4 \mathrm{~mm}, \mathrm{FSR}=0.12 \mathrm{~nm}$. Bottom: etalon 2 transmission, $d=1.1 \mathrm{~mm}, \mathrm{FSR}=0.15 \mathrm{~nm}$.

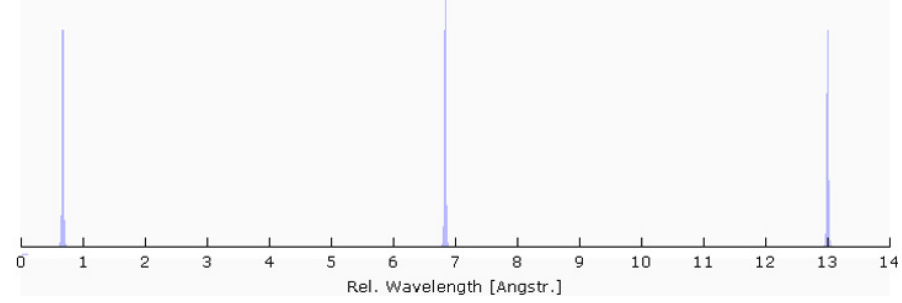

Fig. 5. Double etalon spectral transmission.

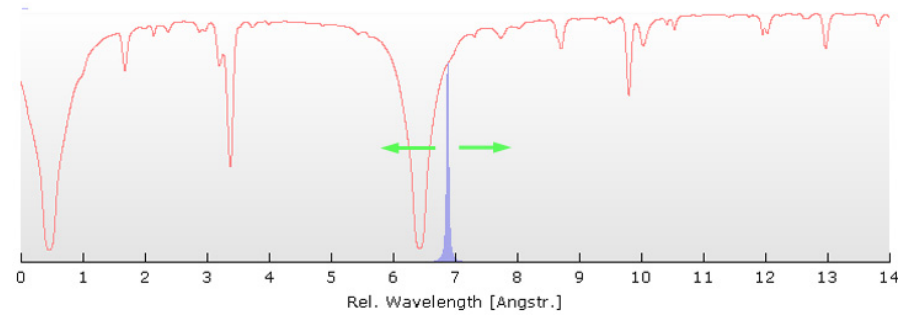

Fig. 6. Fabry-Perot line scanning.

repeatedly. The ability to do this at a high cadence will largely depend on the ability to shift and reposition filter mounts in a fast and convenient manner.

The traditional approach to this task has been to fit narrowband prefilters into a filter wheel and rotate these filters successively through the instrument's main beam. The inherent limitations are low angular acceleration and FOV stability issues, which tend to become even more challenging when trying to increase the number of prefilters.

To avoid these problems we skipped this classical approach in favor of a matrix-type solution that not only accomodates a larger number of filters but also offers sub-micron positional accuracy at high displacement speed (Fig. 7). A dual stepper drive carries out the computer-controlled filter shifting. Filter changes are completed within two seconds. The Cartesian geometry effectively removes the mechanical obstacles of the off-axis rotational geometry (Staiger 2011).

Sixteen filters from the VTT stock may be mounted into a four-by-four grid pattern. Individual mounts may be removed easily from the background matrix for fast filter changes. Tilting may be adjusted individually for each filter in two angular axes with respect to the incoming light beam. Reflected light from the filter surface serves as a visual reference to achieve resolutions of below $0.2 \mathrm{deg}$ per axis. This allows fitting spectral lines of 


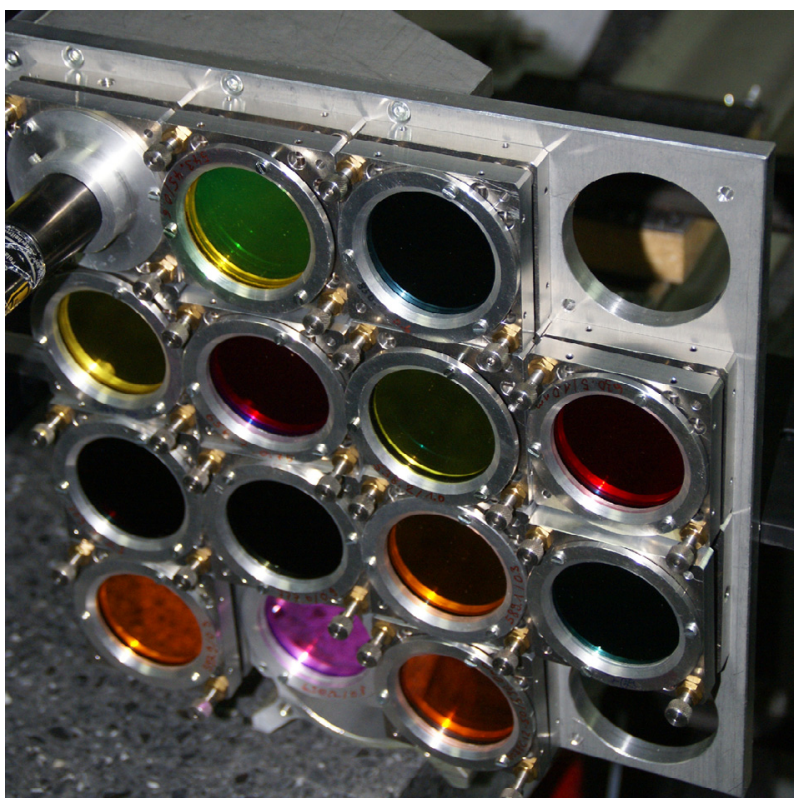

Fig. 7. Matrix filter shifter ready for observation.

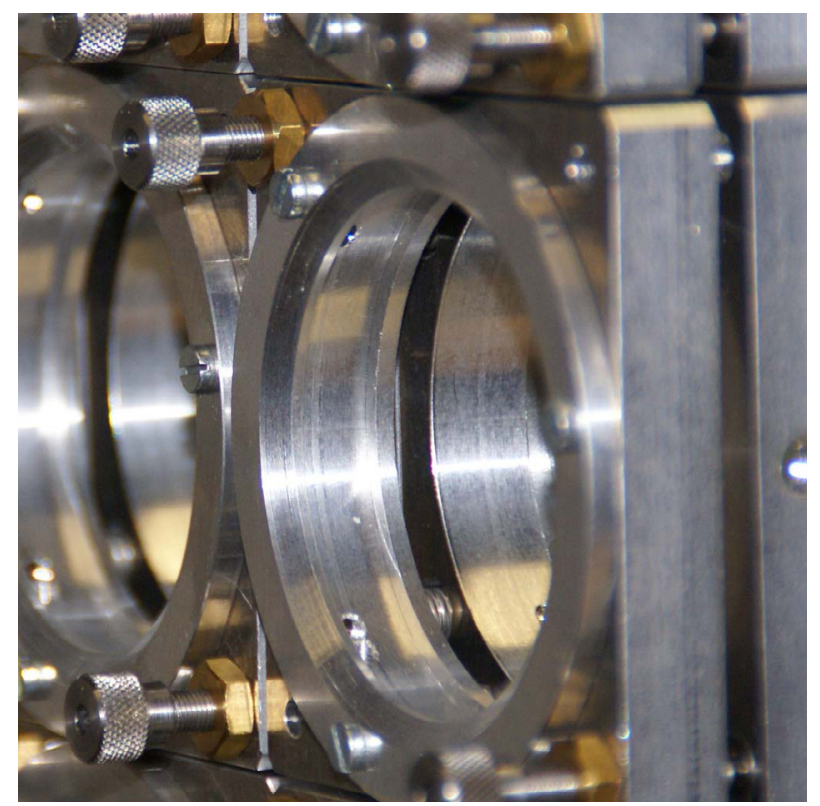

Fig. 8. Individual filter mount.

interest symmetrically into the filter's transmission curve with respect to the transmission maximum. Figure 8 shows some mechanical details of the spring-balanced gimbal mounts.

\section{Software}

\subsection{Overview}

Preparing an etalon-based spectrometer may consume a significant proportion of an observing time slot. Obviously this problem will intensify when the number of spectral lines increases. A software package consisting of the following tools has been developed to adapt to this situation:

- embedded process simulation;

- semiautomatic setup;

- quicklook and pre-processing.

\subsection{Embedded process simulation}

A numerical model of a collimated beam spectrometer has been integrated into the software. It contains a dual etalon model with full handling functionality. Model access is identical to real instrument access. Switching between modes is by mouse click. The development was done with the following goals in mind.

- The model environment creates a transparent picture of device operation.

- The impact of the measurement process itself onto the final results may be investigated with the help of artificial velocity fields.

- With system overhead being identical for simulation-mode and real-mode, stability issues may be resolved at an early stage of development.

- Observational setups may be prepared offline. Users may become familiar with multiline operations prior to observations.

The model has the following properties:

- Spectrum: Spectral input to the model instrument is provided by the Liege Spectral Atlas (1973). The wavelength range of between $480 \mathrm{~nm}$ and $900 \mathrm{~nm}$ fully covers the etalon's transmission bandpass between $560 \mathrm{~nm}$ to $860 \mathrm{~nm}$. Wavelength resolution is at $0.02 \mathrm{~nm}$. Simulation box size currently stands at $2 \mathrm{~nm}$ and may be extended for future applications. Wavelength shifts from model velocity fields may be directly applied to line profiles. This allows creating realistically distorted profiles that display differential wavelength shifts with absorption depth.

- Etalons: the Fabry-Perot etalons are treated as a set of dual plates of a given average (zero order) spacing and predefined reflectivity. The shape of the Airy function and the free spectral range may be derived from these data and the predefined finesse values.

- Piezo operations: currently the model allows only for the simultaneous piston type elongation of the triple piezo stack but not yet for any kind of relative plate tilting. The change in finesse as caused by such an asymmetric piezo configuration is instead taken care of by a broadened Lorentzian profile (enhanced FWHM) folded onto the etalon's transmission pattern. Wavelength scanning is simulated by varying piezo elongation, hence etalon spacing.

- Filter transmission: at this time we have modeled 20 of the approximately 100 narrowband prefilters available at the VTT. The filter transmission curves are approximated by a Gaussian function of given FWHM overlaying the selected spectral band.

- Velocity fields: artificial velocity fields may be fed into the simulation pipeline. Currently these fields are limited to basic geometrical patterns like rectangular or circular piston flows or monomodal oscillations. It is already possible nethertheless to implement amplitude dependencies with height and to overlay different velocity patterns. In the future, realistic P-mode wave fields may be inferred.

The velocity simulation box extends from the continuum level to a height level of $1500 \mathrm{~km}$. At a spacing of $10 \mathrm{~km}$ this will result in 150 vertical grid points above the solar surface. The horizontal points represent the $2 \mathrm{D}$ observation field and are arranged as a square $512 \times 512$ matrix that maps the CCD pixel pattern to the observation area. Spacing is $0.2^{\prime \prime}$ in both directions. For every instant of time a box of size $512 \times 512 \times 150$ will thus represent the instantaneous velocity distribution above the field of view continuum level. 
The velocities are to be converted into line shifts. For each spectral line under investigation and for every instant of time, conversion will result in a box of size $512 \times 512 \times 100$, where the vertical axis is now a wavelength axis at $0.0002 \mathrm{~nm}$ resolution. The grid points along the vertical axis will contain the instantaneous line profile for every observational pixel. The model is capable of creating line shape asymmetries and line shape distorstions as caused by amplitude changes with height or by propagation phenomena.

- Dispersion: the spectral box as emerging from the velocityto-line-shift conversion may be used to extract the instantaneous CCD image during a simulated FPI run in a very convenient way.

Cutting horizontally through the box at the wavelength value representing the current FPI transmission will directly establish the desired intensity map as seen by the simulated CCD camera.

This is only valid as long as any dispersion effects across the field of view may be neglected, as is the case for telecentric instrument layouts. The collimated beam layout as used in our instrument setup instead requires extrapolating intensities along a curved surface that takes the angular, rotationally symmetric dependency of the transmitted wavelenghts with respect to the center beam into account. In this way the simulated CCD chip will see velocity contributions from different heights of the solar atmosphere like in real-world observations.

We hope to further refine the model and publish performance details in the future.

- CCD: temporal and recording characteristic of the CCD detector were modelled according to experimentally derived values with the DALSA camera.

- Drives: the telescope drives and the filter shifter drives were modelled with the same acceleration and speed characteristics as the real-world drives.

- GUI: simulation screens and observation screens are identical.

- Telescope: the spectroscopic instrument model is directly coupled to the already existing VTT telescope control model. The analysis of the combined system will be used to identify artificial contributions to the observational results from the measurement process.

- Seeing: currently the the influence of changing atmospheric conditions has not yet been implemented in the model. In a future version we plan to include image distorstion and blurring.

The model software as described here is ready to run on any notebook computer.

\subsection{Semiautomatic setup}

The following interactive screens will assist the user in system setup:

- finesse adjustment assistant;

- stepping-ratio auto calibration;

- Piezo scan-lock servo;

- NBF placement assistant;

- spectral atlas line selector;

- scan parameters fast setup;

- Doppler calibration;

- storage usage optimization assistant;

- scansets library.
Table 1. Scanned spectral lines.

\begin{tabular}{lcc}
\hline \hline Wavelength [nm] & Element & Scansteps \\
\hline 517.2 & $\mathrm{Mg}_{\mathrm{I}}$ & 20 \\
538.0 & $\mathrm{C}_{\mathrm{I}}$ & 15 \\
538.1 & $\mathrm{Fe}_{\mathrm{I}}$ & 15 \\
538.2 & $\mathrm{Ti}_{\mathrm{I}}$ & 15 \\
543.4 & $\mathrm{Fe}_{\mathrm{I}}$ & 10 \\
557.6 & $\mathrm{Fe}_{\mathrm{I}}$ & 20 \\
589.0 & $\mathrm{Na} \mathrm{D}_{2}$ & 30 \\
589.6 & $\mathrm{Na} \mathrm{D}_{\mathrm{I}}$ & 30 \\
630.1 & $\mathrm{Fe}_{\mathrm{I}}$ & 20 \\
630.15 & $\mathrm{Telluric}_{\mathrm{l}}$ & 15 \\
630.2 & $\mathrm{Fe}_{\mathrm{I}}$ & 15 \\
632.8 & $\mathrm{He}-\mathrm{Ne} \mathrm{Laser}_{2}$ & 15 \\
656.3 & $\mathrm{H}_{\alpha}$ & 50 \\
709.1 & $\mathrm{Fe}_{\mathrm{I}}$ & 20 \\
777.1 & $\mathrm{Fe}_{\mathrm{I}}$ & 20 \\
777.2 & $\mathrm{Fe}_{\mathrm{I}}$ & 10 \\
\hline
\end{tabular}

All activities are based on mouse clicks. File handling does not require user interaction. These tools should limit the time span required to adjust a single spectral line to a few minutes. All parameters of an active measurement are stored within a quickaccess storage. Restarting an observation on a new day or after a system failure will thus require just a few mouse clicks.

\subsection{Preprocessing}

Instant quality judgment at the end of an observation with the following tools may help to detect runtime problems at an early stage and thus avoid follow-up loss of observing time.

- Data quick look: recorded data are instantly available for visualization without the need to leave the operating environment. Data may be randomly accessed. Overview movies are instantly available with the option of zooming into regions of interest.

- Spectral analysis: low-spatial-, high-temporal resolution spectral analysis may be carried out instantly at the end of a run. This module operates on a downscaled set of image data $(64 * 64$ instead of $512 * 512)$ created in parallel to the master data set. In this mode it takes appr. ten seconds to get a preliminary analysis of a ten-hour data run. Part of this package is a ring diagram queue as first established by Hill (1988). In its full resolution version, it will be used to analyze wave propagation and wave damping above the solar surface.

\section{Test results}

\subsection{Spectral lines}

During an observational campaign at the VTT in July 2009 we were able to demonstrate the functionality of the new instrument. Table 1 gives an overview over the spectral lines observed simultaneously.

The spectral lines were selected with the intention of covering a broad range of formation heights extending from the low photosphere $(538.0 \mathrm{~nm}, \mathrm{C}$ I) into the middle chromospheres (656.3 $\mathrm{nm}, \mathrm{H} \alpha)$. The number of scansteps varied with line strength. Besides solar lines, a telluric line and a laser line were included in order to monitor system stability.

Weather conditions during the test campaign allowed for just one observing run. Data were collected over a period of four 


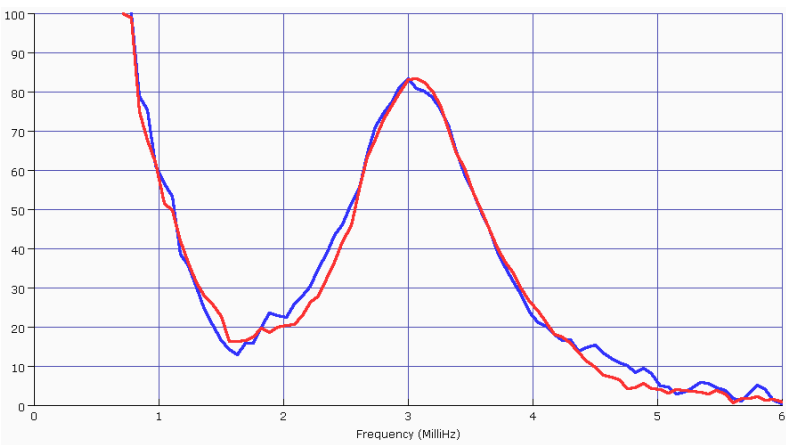

Fig. 9. Linear powerspectra of photospheric lines $557.6 \mathrm{~nm}, \mathrm{Fe}$ I (red) and $630.2 \mathrm{~nm}, \mathrm{Fe} \mathrm{I}$ (blue).

hours. After that cloudy skies prevailed. Despite these conditions the recording run was extended to ten hours duration to test the long-term software stability.

Although a run duration of four hours cannot be regarded as sufficient to analyze oscillations to our desired level of quality, some of the diagrams derived from this measurement will give an indication of future instrument use and capabilities.

\subsection{Photosphere}

Two of the recorded spectral lines $557.6 \mathrm{~nm}(\mathrm{Fe} \mathrm{I})$ and $630.2 \mathrm{~nm}$ (Fe I) have their origin in the photosphere with closely spaced formation heights of appr. $290 \mathrm{~km}$ and $310 \mathrm{~km}$ above the continuum level (Schleicher priv. comm.). This close neighborhood seems to be confirmed by the linear spectra almost matching in the vicinity of the five-minute oscillations peak around 3 milliHz (Fig. 9). Both curves were calibrated to equal peak maximum. Individual p-modes were smoothed out by running means and by accumulating individual pixel spectra. The 557.6 peak region (red) is slightly shifted towards higher frequencies by 0.05 milliHz with respect to the 630.2 spectrum. We see this as an indication that the new instrument will be capable of resolving subtle power details.

\subsection{Transition zone photosphere - chromosphere}

Figure 10 displays two linear power spectra representing the upper photosphere (543.4 nm, Fe I, blue curve) and the low chromosphere (656.3 $\mathrm{nm}, \mathrm{H} \alpha$, red curve). In both spectra the five-minute oscillations show up prominently near $3 \mathrm{milliHz}$, but the peak maxima are now separated by appr. 0.2 milliHz. Additionally the chromospheric line displays a strong power contribution near $5 \mathrm{milliHz}$, that may be linked to the emergence of three-minute oscillations. The $\mathrm{H} \alpha$ spectrum seems to be more rugged and less regular than the photospheric counterpart. This may be attributed to the stronger dynamics in the chromosphere and to a more complicated and broader line formation contribution function. By recording over extended times and observing other chromospheric lines of interest (infrared triplet), we hope to be able to investigate the formation of the three-minute oscillations with the new spectrometer in more detail.

\subsection{Peak oscillatory power shift}

The upper sections of three five-minute power peaks representing the deep photosphere $(538.0 \mathrm{~nm}, \mathrm{C} \mathrm{I})$, the transition region from photosphere to chromosphere $(589.0 \mathrm{~nm}, \mathrm{Na} \mathrm{I})$ and the chromosphere $(656.3 \mathrm{~nm}, \mathrm{H} \alpha)$ are shown in Fig. 11. The diagram

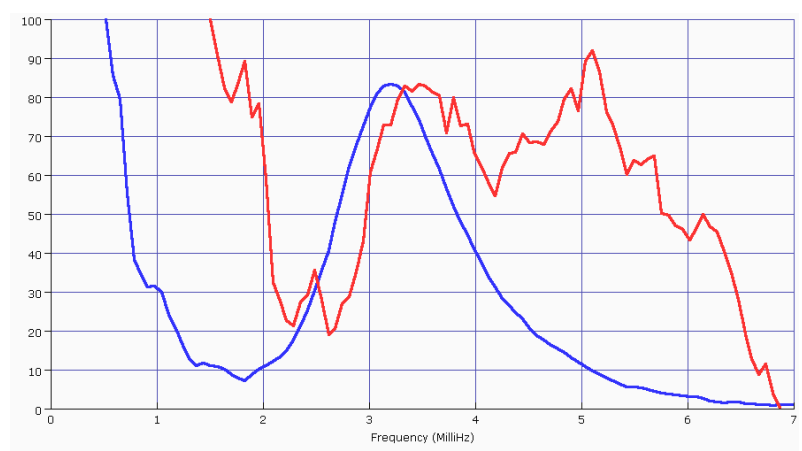

Fig. 10. Linear powerspectra $543.4 \mathrm{~nm}, \mathrm{Fe}$ I (blue) and $656.3 \mathrm{~nm}, \mathrm{H} \alpha$ (red).

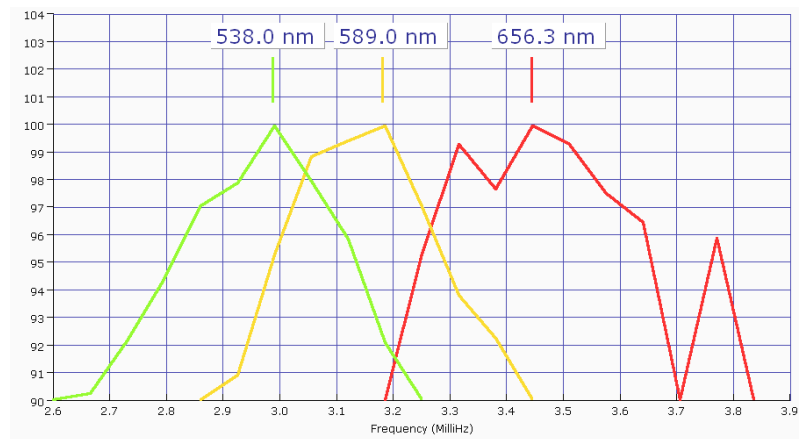

Fig. 11. Linear powerspectra $538.0 \mathrm{~nm}, \mathrm{C}$ I (green), $589.0 \mathrm{~nm}, \mathrm{Na}$ I (yellow), and $656.3 \mathrm{~nm}, \mathrm{H} \alpha$ (red).

reveals a change in peak frequency from 2.95 milliHz $(538.0 \mathrm{~nm})$ towards $3.45 \mathrm{milliHz}(656.3 \mathrm{~nm})$.

This shift may be related to frequency-dependent wave damping with an estimated shift rate of appr. 0.04 milliHz per $100 \mathrm{~km}$ height increase in line formation. We assume that a more thorough analysis will reveal more detailed information about this damping process and line formation heights.

\subsection{Multiple diagnostic diagrams}

Figure 12 (following page) shows a screen dump of eigth of the full set of 16 diagnostic diagrams as created by the spectral analysis tool shortly after the end of the test run. P-mode ridges may be detected at any level from the low photosphere (upper left, $538.0 \mathrm{~nm}$ ) to the chromosphere (lower right, $656.3 \mathrm{~nm}$ ). From these data it seems to be possible with the new instrument to trace phase- and amplitude properties of individual p-modes up through the solar atmosphere at unprecedented vertical resolution.

\subsection{Multiple ring diagrams}

Figure 13 depicts horizontal cuts through the 3D-spectra of the eight spectral lines near the frequency of $3.3 \mathrm{milliHz}$. All lines show some form of circularly arranged power to be expected from $\mathrm{p}$-mode ring diagrams. We plan to use such diagrams to investigate wave spread characteristics around activity regions simultaneously at different height levels. We expect even better quality data in the future, as these spectra have been derived from raw data without any flatfield-, dark current-, or imagemovement corrections. 


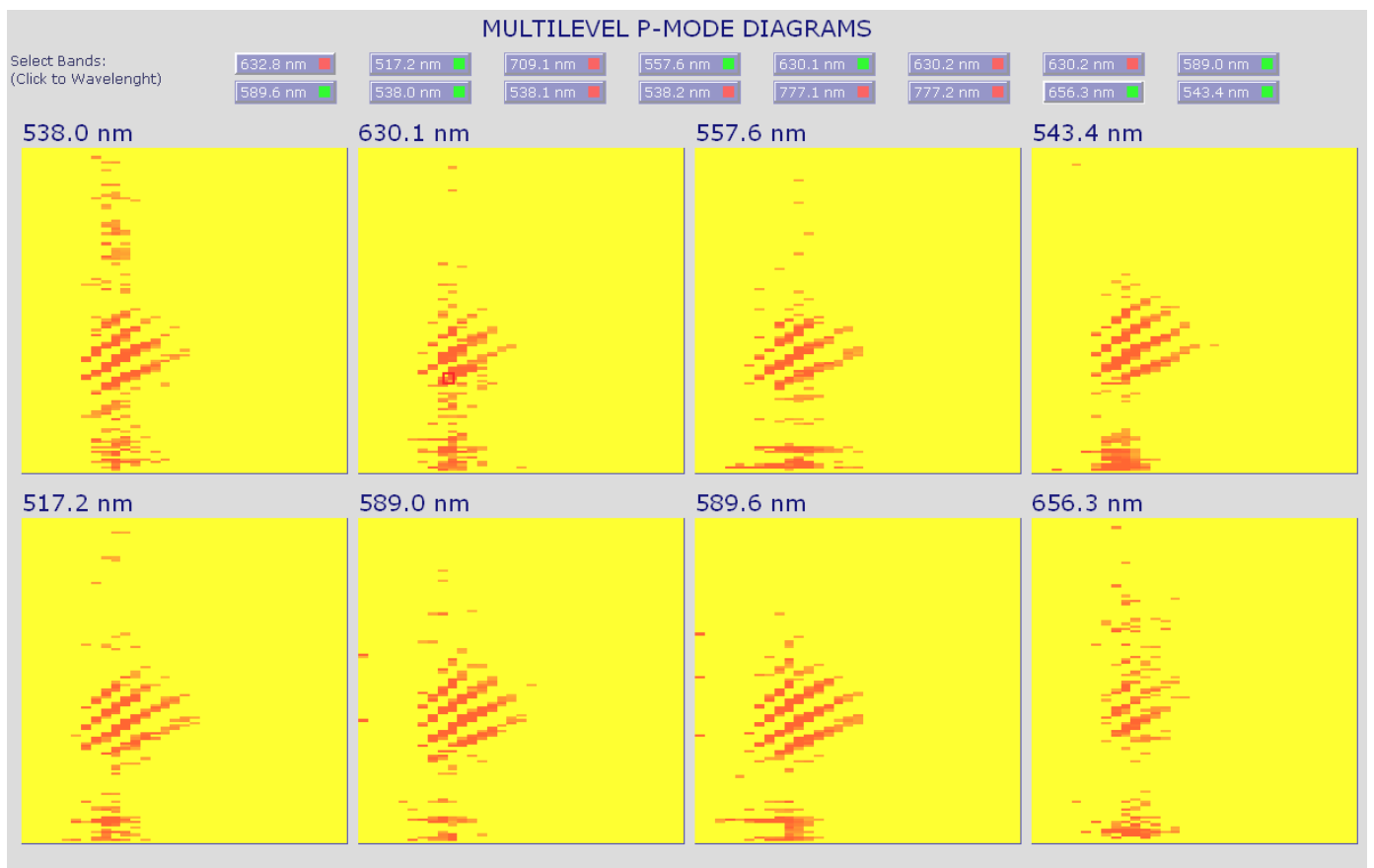

Fig. 12. Screenshot of multiple diagnostic diagrams.

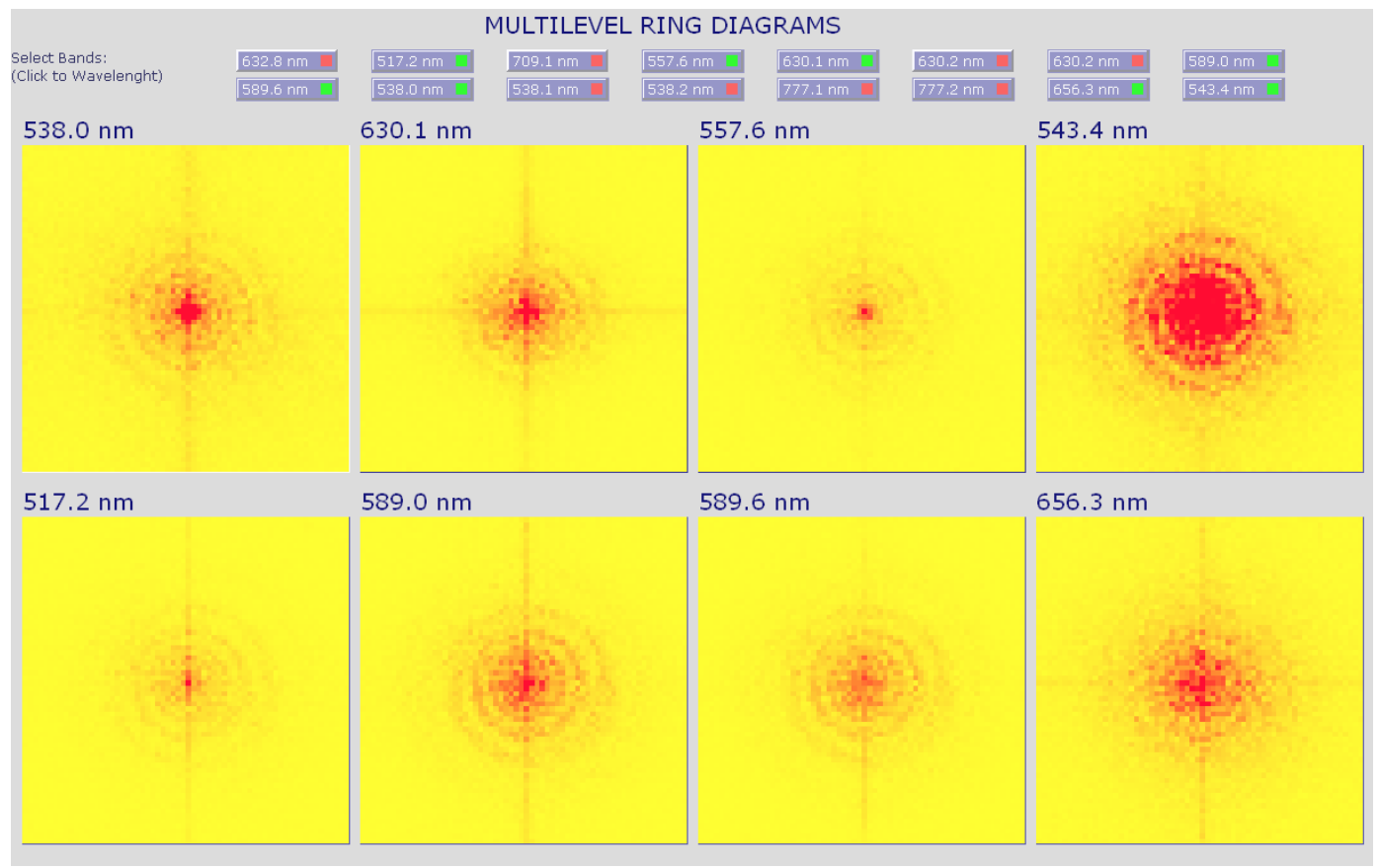

Fig. 13. Screenshot of multiple ring diagrams.

\section{Conclusions}

We have developed a dual-etalon spectrometer specifically designed for the helioseismological mapping of solar atmospheric velocities. During a test run at the VTT, we were able to demonstrate that the instrument allows for simultaneous observations at multiple height levels.

We designed a new matrix type filter shifting unit that allows at a higher displacement speed more narrowband prefilters to be accomodated than previously possible. The number of spectral lines currently stands at 16 lines per minute with room left for improvement. The software allows for observing runs of up to $12 \mathrm{~h}$ duration. The field of view is 100 by 100 arcsecs.

For the first time for an instrument of this type, the operating software has been designed as an integrated package consisting of process simulation, data recording and data preprocessing. This integrated approach allows us to better understand the impact of the measurement process itself onto the results, thus defining validity bounds for any scientific conclusions.

The simulation package allows users to become familiar with system-handling properties prior to observing. The instrument model was the core software element from which the real-world etalon-handling procedures evolved. 
The data processing software contains a ring diagram pipeline. A quick-look package allows judging the spectroscopic quality of a measurement immediately after run termination.

Future scientific applications will set their focus on:

- energy flux analysis;

- wave mode conversion;

- magnetic canopy mapping;

- short-term event detection (flare response).

Acknowledgements. The author wants to express his gratitude to Prof. Franz Kneer (IAG Göttingen) for his generous permission to use the two GFPI-etalons during the VTT-tests. This permission was essential for the project to become reality. The strong support of Prof. Svetlana Berdyugina from KIS made continuing the project possible after the GFPI etalons were no longer available. The skills and superb craftsmanship of KIS mechanical engineer Thomas Sonner were the basis for the construction and successful performance of the filter-matrix shifter, which is the opto-mechanical core element of this instrument. The continuous interest of the head of the KIS Helioseismology Group Dr. Markus Roth proved to be an especially valuable element with the ongoing development of this instrument. I can always count on the fast support of electronics engineer M. Weissschädel, systems manager M. Knobloch, and telescope operations engineer T. Keller. This is especially important in the context of VTT technical functionality. We would like to thank Dr. Klaus Puschmann (then IAG Göttingen, now AIP, Potsdam) for introducing us to the original GFPI-instrument before this project started. During the initial phase of the development, Dr. Nazaret Bello Gonzalez (then IAG, now KIS Freiburg) provided much esteemed backup support with the GFPI in case the first helioseismological tests with the new instrument had failed. We were very thankful for this. We want to thank Dr. Hubertus Wöhl (KIS) for his support during the first scientific tests and for his always friendly attitude as a long-time colleague. The author wishes to acknowledge the support received from the European Helio- and Asteroseismology Network (HELAS) as funded by the European Commission's Sixth Framework Program. Finally we want to thank the anonymous referee for some very helpful comments on the embedded process simulation procedures and Joli Adams for fast and immediate language editing.

\section{References}

Bendlin, C., Volkmer, R., \& Kneer, F. 1992, A\&A, 257, 817

Bonaccini, D., Cavallini, F., Ceppatelli, G., \& Righini, A. 1989, A\&A, 217, 386

Cavallini, F. 2006, Sol. Phys., 236, 415

Cavallini, F., Ceppatelli, G., Righini, A., \& Barletti, R. 1979, A\&A, 85, 255

Delbouille, L., Neven, L., Roland, G. 1973, Université de Liège

Hill, F. 1988, ApJ, 333, 996

Kentischer, T., Schmidt, W., Sigwarth, M., \& Uexkll, M. 1998, A\&A, 340, 569

Loughhead, R. E., Bray, R. J., \& Brown, N. 1978, Ap. Opt., 71, 415

Mack, J. E., McNutt, D. P., Roesler, F. L., \& Chabbal, R. 1963, Ap. Opt., 2, 873

Puschmann, K. G., Kneer, F., Seelemann, T., \& Wittmann, A. 2006, A\&A, 451, 1151

Ramsay, J. V., Kobler, H., \& Mugridge, E. G. V. 1970, Sol. Phys., 12, 492

Reardon, K., Cauzzi, G., Teriaca, L., Pitterle, M., \& Curdt, W. 2008, 12th Europ. Sol. Phys. Meeting, Freiburg

Righini, A., Cavallini, F., \& Reardon, K. P. 2010, A\&A, 515, A85

Scharmer, G. B. 2006, A\&A, 447, 1111

Scharmer, G. B., Narayan, G., Hilberg, T., et al. 2008, ApJ. 689, L69

Staiger, J. 2011, JPCS, 271

Vecchio, A., Cauzzi, G. W., \& Reardon, K. 2009, A\&A, 494, 269 